\title{
Improving Tactile Map Usability through 3D Printing Techniques: An Experiment with New Tactile Symbols
}

\author{
Jaume Gual Ortí, Marina Puyuelo Cazorla ${ }^{2}$ and Joaquim Lloveras Macia ${ }^{3}$ \\ ${ }^{1}$ Universitat Jaume I, Castellón, Spain. ${ }^{2}$ Universitat Politècnica de València, València, Spain. ${ }^{3}$ Universitat Politècnica \\ de Catalunya, Barcelona, Spain \\ Email: jgual@esid.uji.es
}

This paper shows an experiment with tactile maps designed for visually impaired persons. Tests were carried out on a tactile map produced with $3 D$ printing and including a new type of tactile symbols, volumetric symbols $(3 D)$. These symbols are localized faster than conventional flat relief symbols, with the same error rate, an improvement in the use of these tactile devices. Moreover, following tests, differences were found between types of participants with blind participants generally carrying out the proposed tasks better than the rest of users.

4 Keywords:

\section{OBJECTIVE}

The objective of this paper is to determine whether there are differences in the use of tactile maps when the symbols used have different shape dimensions, comparing twodimensional (2D-flat relief) and three-dimensional characters (3D-volumetric).

In addition, this paper tries to determine whether there are differences in the results depending on the different profiles of the participants: sighted, partially sighted or blind persons.

\section{INTRODUCTION}

\begin{abstract}
Maps and tactile symbols
Tangible graphics are devices often used to teach graphic information to the blind or visually impaired, by translating graphic information into the sense of touch. Tactile maps are a specific type of tangible graphic, although there are also different types of maps, as classified by Polly Edman: Mobility maps, Topological Maps, Orientation Maps, General Reference Maps (Edman, 1992). These maps generally use the same resources as visual versions when conveying information, including symbols, labels and legends. Although the lower precision of the sense of touch in relation to the sense of sight must be taken into account when designing tactile maps, this fact results in a need for tactile versions to be simple and display less and more synthesized information than visual maps. There are several
\end{abstract}

books and articles which provide recommendations to facilitate their design (Goodrick, 1987; Jehoel et al., 2006; Trevelyan, 1987; Rowell and Ungar, 2003a; Wiener et al., 2010).

In addition, three elements are usually used to design and produce tactile maps: point, linear and areal elements (Amick et al., 2002; Edman, 1992), that is to say, the same elements used in graphic design in the visual domain. Thus, a tactile translation does not usually employ all the possibilities afforded by 3D shapes ( $Z$ axis). In this regard, a relief map is not a product that belongs only in the $2 \mathrm{D}$ world.

Generally, the selection of tactile symbols for use on tactile maps ought to take into account various factors and recommendations for easy reading and tactile discrimination, among other aspects (Edman, 1992; Wiener et al., 2010). Some tactile symbols have already been evaluated in other experimental works (McCallum et al., 2006; Rener, 1993; Rowell and Ungar, 2003b; Nolan, 1971; Lambert and Lederman, 1989), but the symbols analysed are mainly two-dimensional, and consequently of constant height, showing these shapes in flat relief or with little variation in their elevation $(2.5 \mathrm{D})$. Some studies suggest a minimum relief height (Jehoel et al., 2006), but it is also important to contextualize the symbols in a real situation, not just as stand-alone elements on separate test cards, in order to analyse the elevation effect along with the rest of the elements of the map (Jehoel et al., 2005).

In this study, researchers chose a tactile map of an urban area on which to apply volumetric tactile symbols (basic figures) simulating a real context to observe whether it is 
possible to improve the use of tactile maps, taking into account the fact that $3 \mathrm{D}$ shapes such as spheres or cubes are difficult to confuse with 2D ones (points, lines and areas). Previous work (Gual et al., 2011, 2012) was used to determine the selection of the volumetric tactile symbols used in this study.

This prompts the question as to why other simple geometries such as basic prisms that can be easily recognized through touch are not used as tactile symbols (Gual et al., 2012) or why these geometries, which are generally well known, cannot be included on a tactile map with the current set of symbols.

\section{Systems of production}

These issues become more important if one takes into account the improvements in production techniques such as the emerging trend of Rapid Manufacturing or Rapid Prototyping (Chua et al., 2003; Zhang, 1996), over the traditional systems currently used to produce tactile maps: microencapsulation (also called swell paper) and thermoforming (Rowell and Ungar, 2003c). Microencapsulation represents the paradigm of direct use of $2 \mathrm{D}$ design elements: points, lines and areas, and is a system which can only produce monochrome tactile maps. In contrast, 3D printing can produce complex polychrome geometries, which means that it is capable of combining visual and tactile features to generate maps within the perspective of Inclusive Design (Keates and Clarkson, 2003).

The two techniques for producing tactile maps used in this study, microencapsulation and $3 \mathrm{D}$ printing, have a fundamental similarity as they are both used in the production of small series or unique pieces. The $3 \mathrm{D}$ models produced through Rapid Prototyping can also be used as master pieces in the thermoforming process. However, there are significant differences between the two techniques. The first of these is the duration of use, as microencapsulated paper is less resistant to touch degradation than any of the polymers or materials used in Rapid Prototyping techniques. Nevertheless, the swell paper used in the microencapsulated system is flexible and therefore more portable than the physical rigid model of $3 \mathrm{D}$ printing. Finally, 3D printing pieces can be used as evaluation models in the verification stages of the development of new products.

\section{METHODOLOGY}

The methodology used in this study was based on performing tasks with prototypes (time spent on tasks).

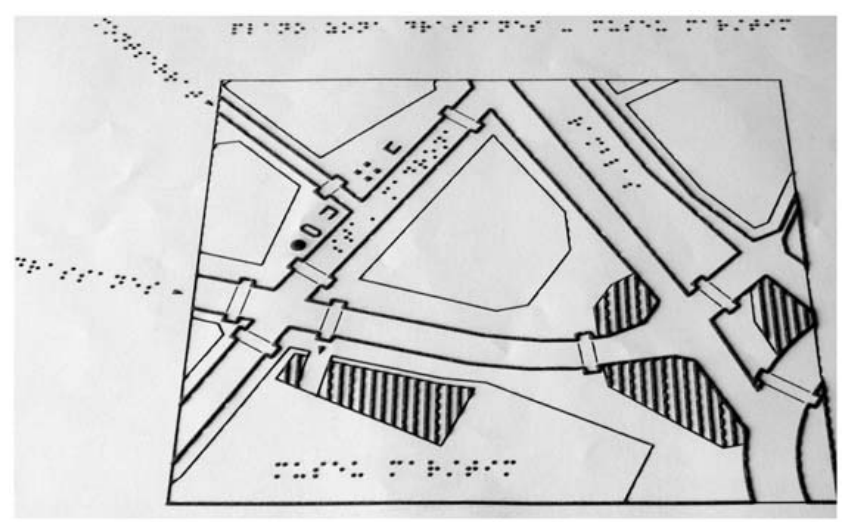

Figure 1. Map A. Microencapsulated

\section{Sample}

The experiment described in this paper was performed with two independent groups of participants: Group 1 and Group 2. The sample consisted of 56 people, 28 per group, with a total mean age of 43.82 (SD: 13.93) years and ages ranging from 20 to 80 years. Group 1 had a mean age of 43.32 (SD: 13.96) years, while Group 2 had a mean age of 44.32 (SD 14.13) years.

Table 1 shows distribution according to the participants' profiles. Sighted and partially sighted participants conducted the experiment blindfolded in order to compare only the tactile perception level between the different user profiles. Blindfolded participants who did not know how to read Braille were assisted verbally to substitute the relief information.

\section{Material used in the study}

Two tactile maps were used in this study, one for each group. The first, Map A, was produced on monochrome microencapsulated (swell paper) (Figure 1), while the second, Map B, was a version of the same map, produced using polychrome 3D Printing (Figure 2).

Both maps represent the same defined area of the city of Barcelona (scale 1:1250), and are identical in size $(219 \times 190 \times 20 \mathrm{~mm})$, element distribution and amount of information represented. Both have a key with six tactile symbols (Figure 3 ) which express the following meanings:

- subway entrance/exit;

- telephone booth;

- kiosk;

- urban lift;

- pedestrian crossing;

- green area.

Table 1. Table of segregation of the sample according to participant profiles

\begin{tabular}{llll}
\hline Participant profile & & & \\
\hline Group & Blind persons & Low vision (blindfolded) & Sighted (blindfolded) \\
\hline Group 1 & 15 & 6 & 7 \\
Group 2 & 15 & 4 & 9 \\
\hline
\end{tabular}






Figure 2. Map B. 3D printing

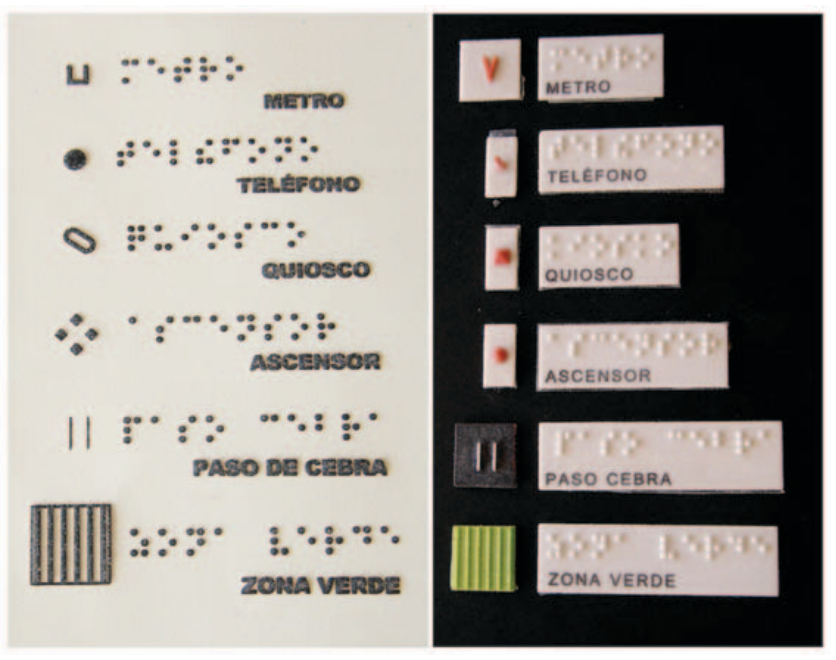

Figure 3. Key of Map A and Map B

Map A

This Map (Figure 1) is configured with typical 2D design elements, points, lines and areas, as well as Braille text to designate the streets. Edman's recommendations were followed when designing this map (Edman, 1992). Relief
Table 2. Summary table with the symbols evaluated and their characteristics depending on map

\begin{tabular}{lll}
\hline Items & Map A & Map B \\
\hline Subway entrance/exit & $2 \mathrm{D}$ & $2 \mathrm{D}$ \\
Telephone booth & $2 \mathrm{D}$ & $3 \mathrm{D}$ \\
Kiosk & $2 \mathrm{D}$ & $3 \mathrm{D}$ \\
Urban lift & $2 \mathrm{D}$ & $3 \mathrm{D}$ \\
\hline
\end{tabular}

lines employ different discriminatable thicknesses for the sense of touch, and lines are used to separate the limits between sidewalk-vehicle transit or sidewalk-buildings. The green area is represented by vertical lines.

Map B

Map B (Figure 2) has formal features typically used for models: there are different levels separating the sidewalk from roads and the ramps are represented with inclines. However, elements of tactile maps are also expressed in the form of lines separating the buildings from the sidewalk with two parallel lines representing pedestrian crossings, or a specific texture used to symbolize the green area. The map includes various items of information represented by volumetric symbols: the telephone box with a cylinder, the kiosk with a pyramid and the urban lift with a cone. Flat relief symbols were used to represent the subway entrance/ exit, the pedestrian crossing and the green area, thus ensuring these items retain similar characteristics to the version of Map A. Figure 4 shows some of the symbols used in Map B. The first from the left in this experiment represents the subway entrance/exit (2D). The rest are the telephone booth, the kiosk and the urban lift respectively (3D).

Tasks and procedure

Two types of task were used in this experiment: counting and localizing symbols in both maps. Participants had to perform these tasks within their respective group: Group 1 was assigned Map A, while Group 2 worked with Map B. The tactile symbols evaluated had the same position and represented the same content on both maps. Their fundamental difference lay in their two-dimensional (2Dflat relief) or three-dimensional (3D-volumetric) nature. Table 2 shows the four tactile symbols evaluated and their shape features on each map.

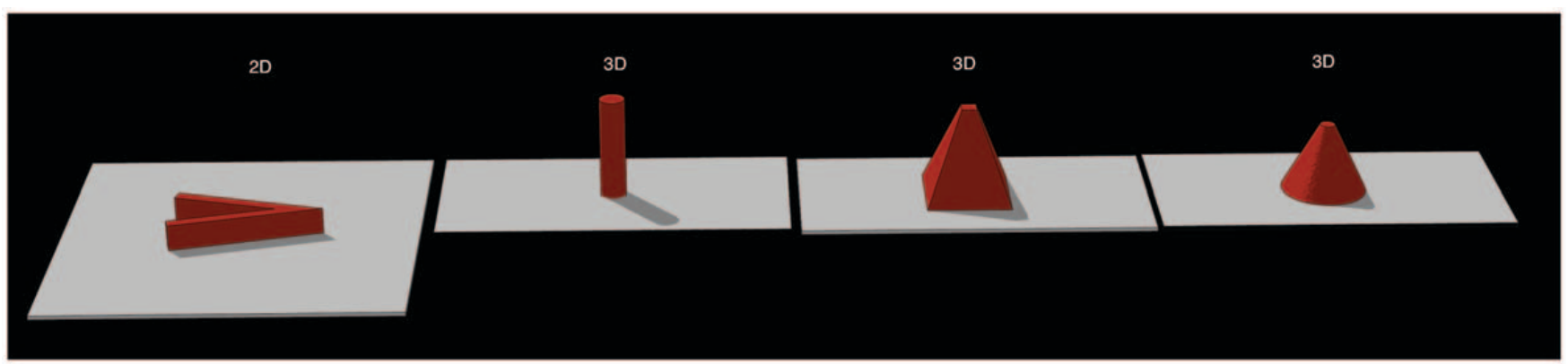

Figure 4. Virtual image of some tactile symbols of Map B: entrance/exit to the subway, telephone booth, kiosk and urban lift, respectively 
Group 1 - Map A - microencapsulated

The internal analysis of each group for the comparison of different participant profiles (Table 7 ) indicated that the blind participants used less time in Group 1 to perform Tasks 2, 3 and 4. By profile, sighted participants spent most time localizing the symbols used. The differences were statistically significant and it is important to mention the high standard deviation, more than double, of sighted participants in relation to the rest.

In addition, the profile analysis pairs indicate that the effect size between blind-sighted participants is high (effect size $=1.10)$, although the power is no greater than 0.8 (power $=0.68$ ). However, in comparing blind-low vision participants, the effect size is very high $($ effect size $=2.03)$ with a power greater than 0.8 (power $=0.98$ ).

Errors within Group 1 (Table 8) show a similar pattern among all participants. Blind participants performed fewer errors, while the sighted made the highest number of errors. However, in terms of statistics the differences obtained cannot be considered significant, as all data range between 0.42 and 0.48 , almost identical.

\section{Group 2 - Map B - 3D printing}

Data from Group 2 (Table 9) show that blind participants used the least time to perform Tasks 2, 3 and 4. Sighted participants took the longest and did so with a Standard Deviation at least double more than that of the rest. In Group 2, all participants performed at least one of Tasks 2, 3 and 4 without errors, with the $N$ (valid) being the total of the sample used.

On the other hand, the pair analysis of the various participant profiles revealed significant differences between

Table 5. Mean, standard deviation, $P$-value, effect size and power of time spent on the location of symbols (Tasks 2,3 and 4) depending on the type of map

\begin{tabular}{llcc}
\hline & $N($ valid $)$ & Mean & SD \\
\hline Group 1 & 26 & 52.75 & 45.92 \\
Group 2 & 28 & 21.50 & 26.46 \\
Mann-Whitney test & $P=0.001$; effect size $=0.83$; power $=0.90$ \\
\hline
\end{tabular}

Table 6. Mean, standard deviation, $P$-value, effect size and power of errors in both groups for Tasks 2, 3 and 4

\begin{tabular}{llll}
\hline Group & $N$ & Mean & SD \\
\hline Group 1 & 28 & 0.44 & 0.34 \\
Group 2 & 28 & 0.23 & 0.30 \\
Mann-Whitney test & $P=0.016 ;$ effect size $=0.67 ;$ power $=0.91$ \\
\hline
\end{tabular}

Table 7. Mean, standard deviation and $P$-value of Tasks 2,3 and 4 within the group

\begin{tabular}{lccc}
\hline Participants & $N$ (valid) & Mean & SD \\
\hline Blind & 14 & 33.83 & 21.82 \\
Low vision & 6 & 81.22 & 24.80 \\
Sighted & 6 & 84.69 & 61.54 \\
Kruskall-Wallis test & $P=0.023$ & & \\
\hline
\end{tabular}

the blind-sighted pairing with an effect size and high power (effect size $=1.33$, power $=0.91)$. The blind-low vision pair had a medium-high effect size (effect size $=0.75$ ), although the power was less than 0.8 (power $=0.57$ ).

Finally, an analysis of the errors obtained in Tasks 2, 3 and 4 within Group 2 (Table 10) shows that the participant profile with the least number of errors was that of the visually impaired. Blind participants were those who made most errors. Nevertheless, the Kruskall-Wallis test results suggest that differences in errors between participants' profiles are not statistically significant.

\section{Comparison between groups by type of participant}

When comparing both groups by type of participant only, there is a decrease in time and errors for all user profiles within Group 2 (Tables 7-10). After applying the MannWhitney test and calculating the effect size and power, the following information was obtained:

- reduction of the time spent in the case of the blind. They had a $P$-value of 0.001 , an effect size of 1.22 and a power of 0.93 . In the contrast between low-vision participants statistical results were: $P$-value $=0.011$, effect size $=3.04$, power $=0.99$. In the case of the sighted, the $P$-value indicated no difference $(P$-value $=0.272)$, although there was a medium-high effect size (effect size $=0.83$ ), power $=0.422$;

- regarding the reduction of errors, the Mann-Whitney test indicated no statistically significant differences between the types of participants in both groups. Only the low-vision participant group had a large effect size

Table 8. Mean, standard deviation and $P$-value of errors within Group 1

\begin{tabular}{lccc}
\hline Participants & $N$ & Mean & SD \\
\hline Blind & 15 & 0.42 & 0.37 \\
Low vision & 6 & 0.44 & 0.27 \\
Sighted & 7 & 0.48 & 0.38 \\
Kruskall-Wallis test & $P=0.805$ & & \\
\hline
\end{tabular}

Table 9. Mean and standard deviation of time spent performing Tasks 2,3 and 4 with different types of participants within Group 2

\begin{tabular}{lcll}
\hline Participants & $N($ valid $)$ & Mean & SD \\
\hline Blind & 15 & 12.66 & 11.12 \\
Low vision & 4 & 21.50 & 12.51 \\
Sighted & 9 & 44.24 & 31.67 \\
Kruskall-Wallis test & & $P=0.006$ & \\
\hline
\end{tabular}

Table 10. Mean, standard deviation and $P$-value of errors within Group 1

\begin{tabular}{lrll}
\hline Participants & N & Mean & SD \\
\hline Blind & 15 & 0.27 & 0.39 \\
Low vision & 4 & 0.08 & 0.17 \\
Sighted & 9 & 0.22 & 0.29 \\
Kruskall-Wallis test & & $P=0.652$ & \\
\hline
\end{tabular}


(effect $\operatorname{size}=1.60$ ) and a power close to 0.8 (power $=0.71$ ).

\section{DISCUSSION}

In this experiment, we have attempted to see whether there are any differences between two types of maps containing the same characteristics and information and representing the same area of a city of the same size and scale. The fundamental difference between them is that they were produced using different techniques and designed accordingly. Thus, the map produced using $3 \mathrm{D}$ printing (Map B) shows the information mostly using volumetric design elements, including tactile symbols in the form of basic prisms. In contrast, Map A only used graphic design elements, that is, elements with a flat relief: points, lines and areas (Edman, 1992; Amick et al., 2002). For the execution of the experiment, two independent groups of participants conducted specific tasks on both maps in order to detect possible differences between them.

The first aspect to emphasize is Task 1, which consisted in the comparison of two symbols (subway entrance/exit) appearing on both maps with flat relief (2D). No differences were observed in the results of participants, and similar errors were obtained for both groups when dealing with flat relief symbols on the map. In this regard, they are two homogeneous groups.

In the remaining tasks, measurements were carried out on errors and the time spent by each participant localizing the evaluated symbols on the map: Group 1 used Map A with flat relief symbols (2D), while Group 2 used Map B with volumetric symbols (basic prisms - 3D) in the same position as those on Map A. Experimental data indicated significant differences between groups and, therefore, between maps. Participants who used Map B had reduced both the time spent performing tasks and discrimination errors to less than half.

As regards the profile of participants within their respective groups, it should be noted that data included show that in the time spent localizing evaluated symbols, blind participants performed the tasks in significantly less time than other users. This is more or less logical if we consider that these participants are probably the users who are most in contact with tactile devices. Moreover, sighted participants display more erratic behaviour, as shown by standard deviation. However, no significant differences were found in error analysis. As noted in the data collected for different profiles of participants in Group 1, those who used the microencapsulated map obtained a similar pattern of tactile recognition. However, in Group 2, the role was reversed and sighted and particularly low-vision participants performed better.

Furthermore, the cross-analysis between groups and profiles of participants indicated that in all cases concerning time spent on localisation tasks, there was a statistically significant improvement with large effect sizes, except for sighted users whose effect size was medium-high. In the case of errors by groups and participants, the statistical tests indicated no significant differences between the profiles of participants in both groups. However, the contrast between the low vision participants shows a high effect size. It is possible that with a larger sample, these data would become significant.
The key of the time spent and errors reduction of this experiment is based on two major factors in the field of tactile perception. On the one hand, the high contrast height between the volumetric symbols of the map and the rest of the elements, which facilitated the localisation of the symbols on the map, sometimes almost immediately, and on the other hand, the ease of tactile discrimination of basic figures is because these are simple shapes that are easy to recognize through the sense of touch, as in the case of tactile symbols employed in Map B. Both aspects show that the design of tactile maps and symbols can be improved with the use of Rapid Prototyping techniques (Chua et al., 2003), as it allows the production of complex geometries with contrast height, which are difficult to produce using other techniques. The microencapsulated system only produces $2 \mathrm{D}$ elements with a flat relief. Thanks to the use of Rapid Prototyping techniques, it is possible to open an opportunity for the study of simple volumetric shapes that improve the use of tactile maps.

\section{CONCLUSIONS}

After analysing the differences between groups in this study, we can conclude that the use of tactile 3D symbols clearly improves the mean of time of certain tasks such as the localisation of specific symbols on a map. Using this type of symbols in the design of tactile maps reduces the time the user spends to find them in an autonomous way, without verbal assistance, thereby improving their use and reducing possible situations of frustration. Moreover, the average of reading errors for volumetric symbols is similar to the current ones, regardless of the user profile.

Furthermore, blind participants showed significant differences when locating the symbols on either type of map, but this was nevertheless the user profile that performed the proposed tasks quickest.

Further studies should investigate the possibilities that $3 \mathrm{D}$ printing opens up in the field of tactile maps. This technique can be used in the design and production of inclusive maps that can incorporate colour, large and relief text, Braille and volumetric features among other aspects, all of which are of interest in the design of tactile devices.

\section{BIOGRAPHICAL NOTES}

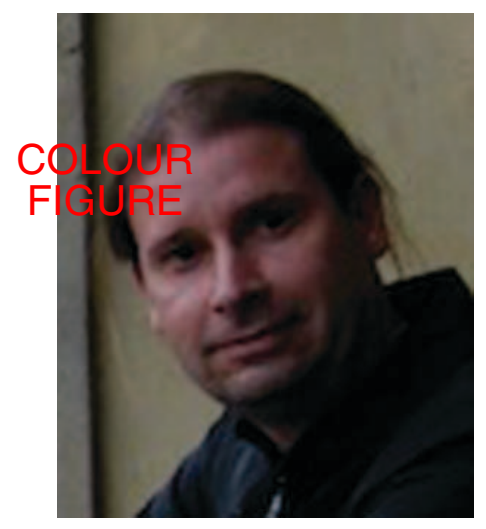

Jaume Gual is a lecturer at Jaume I University, currently working towards a Masters in Product Design Engineering and a doctoral programme in design for collective use and accessibility. His research investigates products for visual impairment, accessibility and inclusive design. 


\section{ACKNOWLEDGEMENTS}

The work reported here is part of the research project 'Estudio y diseño de elementos de orientación, soportes de comunicación y otros accesorios para la mejora de la accesibilidad en distintos ámbitos de interpretación del patrimonio natural $\mathrm{y} / \mathrm{o}$ construidos' supported by the Spanish Ministry of Science and Innovation (project DPI2008-03981/DPI). The authors wish to thank the Centre de Recursos Educatius (Organización Nacional de Ciegos Españoles - ONCE) and the Associació Discapacitat Visual Cataluña $\mathrm{B} 1+\mathrm{B} 2+\mathrm{B} 3$ in Barcelona, as well as the ONCE offices in Castellón, Tarragona and Valencia for supporting this research. Finally, this work has been supported by the Programa de Mobilitat del Personal Investigador de la Universitat Jaume I (E-2010-32) and the Fundació Caixa Castelló-Bancaixa.

\section{REFERENCES}

Amick, N. S., Corcoran, J. M., Hering, S. and Nousanen, D. (2002). Tactile Graphics Kit. Guidebook, American Printing House for the Blind, Inc., Lousville, KY.

Chua, C. K., Leong, K. F. and Lim, C. S. (2003). Rapid Prototyping: Principles and Applications, World Scientific, Hackensack, NJ.

Edman, P. (1992). Tactile Graphics, American Foundation for the Blind, New York.

Goodrick, B. (1987). A map user guide to reading tactual and low vision maps. Division of National Mapping, Department of Resources and Energy. Canberra, Australia.

Gual, J., Puyuelo, M. and Lloveras, J. (2011). 'Three-dimensional tactile symbols. Relief maps for the visually impaired', in Diversity and Unity: Proceedings of IASDR 2011, the 4th World Conference on Design Research, International Association of Societies of Design Research (IASDR) and Delft University of Technology, Faculty of Industrial Design Engineering, Delft, The Netherlands.

Gual, J., Puyuelo, M. and Lloveras, J. (2012). 'Analysis of Volumetric Tactile Symbols Produced with 3D Printing', in ACHI 2012, The
Fifth International Conference on Advances in ComputerHuman Interactions, pp. 60-67, Valencia, Jan 30-Feb 4.

Nolan, C. Y. and Morris, J. E. (1971). Improvement of tactual symbols for blind children. Final Report. Department of Health, Education, and Welfare, US Office of Education, Bureau of Education for the Handicapped and American Printing House for the Blind, Inc. Lousville, KY, USA.

Jehoel, S., McCallum, D., Rowell, J. and Ungar, S. (2006). 'An empirical approach on the design of tactile maps and diagrams: the cognitive tactualization approach', British Journal of Visual Impairment, 24, pp. 67-75.

Jehoel, S., Dinar, S., McCallum, D., Rowell, J. and Ungar, S. (2005). 'A scientific Approach to Tactile Map Design: Minimum Elevation of Tactile Map Symbols', in XXII International Cartographic Conference, A Coruña, Jul 9-16.

Keates, S. and Clarkson, P. J. (2003). Countering Design Exclusion: An Introduction to Inclusive Design, Springer-Verlag, London.

Lambert, L. L. and Lederman, S. L. (1989). 'An evaluation of the legibility and meaningfulness of potential map symbols', Journal of Visual Impairment \& Blindness, 83, pp. 397-403.

McCallum, D., Ungar, S. and Jehoel, S. (2006). 'An evaluation of tactile directional symbols', British Journal of Visual Impairment, 24, pp. 83-92.

Rener, R. (1993). 'Tactile cartography: another view of tactile cartographic symbols', The Cartographic Journal, 30, pp. 195198.

Rowell, J. and Ungar, S. (2003a). 'The world of touch: an international survey of tactile maps. Part 2: design', British Journal of Visual Impairment, 21, pp. 105-110.

Rowell, J. and Ungar, S. (2003b) 'A taxonomy for tactile symbols: creating a useable database for tactile map designers', The Cartographic Journal, 40, pp. 273-276.

Rowell, J. and Ungar, S. (2003c). 'The world of touch: an international survey of tactile maps. Part 1: production', British Journal of Visual Impairment, 21, pp. 98-104.

Trevelyan, S. (1987). Development and assessment of a tactile mobility map for the visually impaired [microform]. National Library of Canada, Ottawa, Ont., Canada.

Wiener, W. R., Blasch, B. B. and Welsh, R. L. (2010) Foundations of orientation and mobility, 3rd ed., American Foundation for the Blind, New York.

Zhang, G., et al. (1996). 'Development of a Rapid Prototyping System for Tactile Graphics Production', in Flexible Automation and Intelligent Manufacturing Conference, Atlanta, GA, May 13-15. 
Authors Queries

Journal: The Cartographic Journal

Paper: 208

Title: Improving Tactile Map Usability through 3D Printing Techniques: An Experiment with New Tactile Symbols

Dear Author

During the preparation of your manuscript for publication, the questions listed below have arisen. Please attend to these matters and return this form with your proof. Many thanks for your assistance

\begin{tabular}{|l|l|l|}
\hline $\begin{array}{l}\text { Query } \\
\text { Reference }\end{array}$ & Query & Remarks \\
\hline 1 & $\begin{array}{l}\text { Please supply the page/paper no. } \\
\text { for Gual et al. (2011). Thanks. }\end{array}$ & \\
\hline 2 & $\begin{array}{l}\text { Please supply the page/paper no. } \\
\text { for Jehoel et al. (2005). Thanks. }\end{array}$ & \\
\hline 3 & $\begin{array}{l}\text { Please supply the full list of } \\
\text { authors' names and page/paper } \\
\text { no. for Zhang et al. (1996). Thanks. }\end{array}$ & \\
\hline 4 & Please supply the keywords. & \\
\hline
\end{tabular}

